\title{
An assessment of simulation methodologies for the analysis of near-field radiation zones related to human exposure
}

\author{
Hugo G. Espinosa and David V. Thiel \\ Centre for Wireless Monitoring and Applications, \\ Griffith University \\ Brisbane (Nathan), Australia \\ h.espinosa@griffith.edu.au
}

\author{
Chris Brindley \\ Corearth Australia Pty Ltd \\ Brisbane, Australia \\ chris.brindley@corearth.com
}

\begin{abstract}
The reliable evaluation of electric and magnetic field exposures with relevant radiofrequency exposure limit standards is important in order to provide protection against known adverse health effects. The evaluation of human exposure levels to radiofrequency, electric and magnetic fields and power density is required for assessment of compliance with technical standards, and it can be conducted by either measurement or numerical calculation. This paper presents a comparison between different computational tools (ProX5 EME, Cylindrical wave model, Flat-zone method and NEC as a reference) for the analysis of zone boundaries for human exposure to radiation from a Yagi-Uda antenna, operating at $450 \mathrm{MHz}$ with a maximum power of $50 \mathrm{~W}$. The results are based on the guidelines for limiting exposure to time-varying electric, magnetic and electromagnetic fields, created by the International Commission on Non-Ionizing Radiation Protection (ICNIRP).
\end{abstract}

Keywords-Near-field radiation; human exposure; timevarying fields, modelling, NEC, ProX5 EME, CENELEC

\section{INTRODUCTION}

In most countries, there are standards that regulate zone boundaries for human exposure to radiofrequency, electric fields, magnetic fields and power flux densities [1]. The evaluation of human exposure levels to those parameters is required for assessment of compliance with technical standards, and it can be performed either by measurement or by numerical calculation [2].

As technical standards continue to evolve for the electromagnetic field modelling, all that are involved in the industry are compelled to continually revisit the basis of calculations. The standard EN 50383:2010: Basic Standard For The Calculation And Measurement For Electromagnetic Field Strength And SAR (Specific Absorption Rate) Related To Human Exposure From Radio Base Stations And Fixed Terminal Stations For Wireless Telecommunications Systems $(110 \mathrm{MHz}-40 \mathrm{GHz})$ [3], requires the validation of commercial software when compared against existing numerical models such as the Method of Moment-based NEC (Numerical Electromagnetic Code) [4].

The new AS2772.2 standard [5] requires that computer simulations include justified uncertainty analysis to be quantified in calculations. This requires the software to be modified to explicitly quantify the uncertainty factors for each calculation, justified against field measurements and other calculation models including NEC.

This study presents an assessment of different computational tools for the analysis of zone boundaries for human exposure from a Yagi-Uda antenna working at $450 \mathrm{MHz}$ with a maximum power of $50 \mathrm{~W}$, through the simulation of the 3D near and far electric and magnetic field patterns, using a commercial software called ProX5 EME and existing tools such as NEC, the Cylindrical wave model and the Flat zone method. Yagi-Uda antennas present a significant challenge as they do not have a recognisable, well defined aperture. An aperture-based analysis would not be conservative due to the position of the active dipole in Yagi-Uda antennas.

The results are based on the ICNIRP reference levels for occupational and general public exposures to time-varying electric and magnetic fields as determined from the unperturbed rms values [6].

\section{Methodology}

\section{A. ICNIRP Reference levels}

The reference levels for human exposure are given for the condition of maximum coupling of the field to the exposed individual, thereby providing maximum protection [6]. Table I summarizes the reference levels for occupational exposure and exposure of the general public. The levels are spatially averaged values over the entire body of the exposed individual and are provided for practical exposure assessment purposes to determine whether the basic restrictions are likely to be exceeded.

TABLE I. ICNIRP REFERENCE LEVELS FOR EXPOSURES TO TIME VARYING ELECTRIC AND MAGNETIC FIELDS (UNPERTURBED RMS VALUES)

\begin{tabular}{|l|c|c|c|c|}
\hline \multicolumn{1}{|c|}{$\begin{array}{c}\text { Reference } \\
\text { level }\end{array}$} & $\begin{array}{c}\text { Freq. } \\
(\boldsymbol{M H z})\end{array}$ & $\begin{array}{c}\text { E-field } \\
(\text { V/m })\end{array}$ & $\begin{array}{c}\boldsymbol{H} \text {-field } \\
(\text { V/m })\end{array}$ & $\begin{array}{c}\text { Power density } \\
\left(\mathbf{W}^{2} \boldsymbol{m}^{2}\right.\end{array}$ \\
\hline $\begin{array}{l}\text { Occupational } \\
\text { exposure }\end{array}$ & $f=450$ & $3 f^{1 / 2}$ & $0.008 f^{1 / 2}$ & $f / 40$ \\
\hline $\begin{array}{l}\text { General public } \\
\text { exposure }\end{array}$ & $f=450$ & $1.375 f^{1 / 2}$ & $0.0037 f^{1 / 2}$ & $f / 200$ \\
\hline
\end{tabular}




\section{B. Antenna selection}

The antenna used for this study consisted of a Yagi-Uda 9element antenna, with a folded dipole as the active element (Fig. 1), working at $450 \mathrm{MHz}$ with a maximum power of $50 \mathrm{~W}$ and a gain of $12 \mathrm{dBd}$. Fig. 1 shows the dimensions of the elements. The length of the elements and the spacing between them were extracted from the RFI manufacturer's technical data sheet for UHF Directional Yagi Antennas, $400-600$ $\mathrm{MHz}$, YB9 Series [7].

The length of the antenna from reflector to last director is $1.42 \mathrm{~m}$, the thickness of the elements is $0.025 \mathrm{~m}$ and the width of the active element (folded dipole) is $0.03 \mathrm{~m}$.

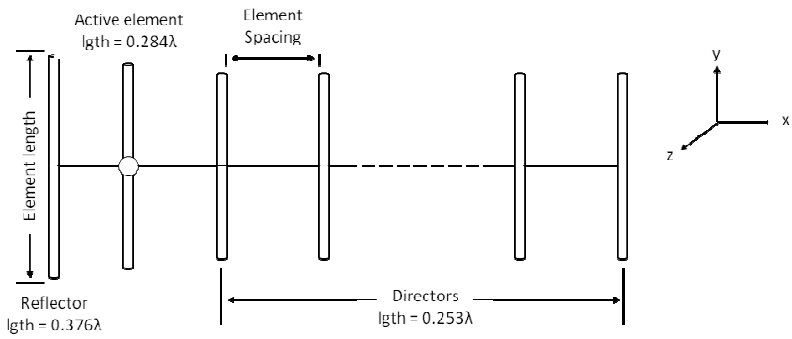

Fig. 1. 9-element Yagi-Uda antenna [7]. The active element is a folded dipole.

\section{Numerical and analytical tools}

Four tools were used for the comparative assessment. The first tool, NEC (Numerical Electromagnetic Code) [4], was used to simulate the $3 \mathrm{D}$ near and far field patterns on a $20 \mathrm{~cm}$ grid bounded by an enclosure of $20 \mathrm{~m} \times 20 \mathrm{~m} \times 20 \mathrm{~m}$. The field exposure reference levels for occupational and general public exposures based on Table I were accurately extracted.

Figs. 2 and 3 show the logarithmic scale for the electric field strength in the $x-z$ and $x-y$ planes, for both occupational and general public exposures, using 50W transmit power. The Yagi-Uda antenna geometrical design is included in the graphics.

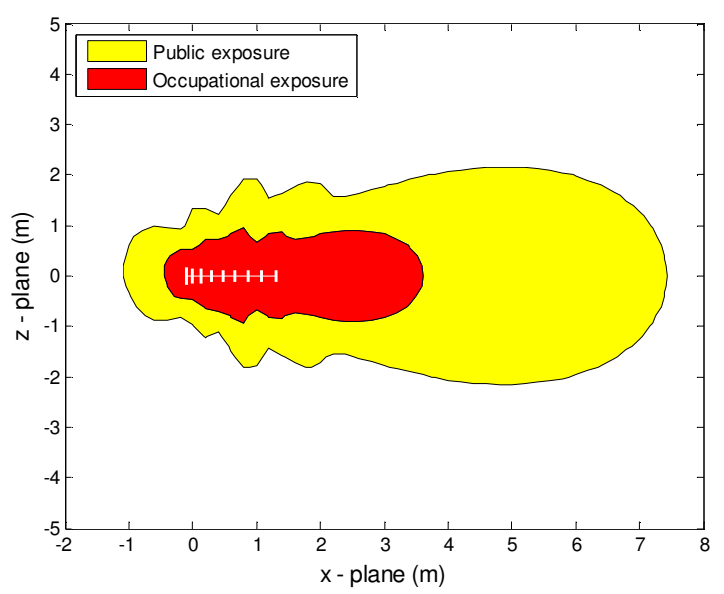

Fig. 2. NEC E-field strength for occupational $(63.6396 \mathrm{~V} / \mathrm{m})$ and general public $(29.1682 \mathrm{~V} / \mathrm{m})$ exposures in $x-z$ plane.

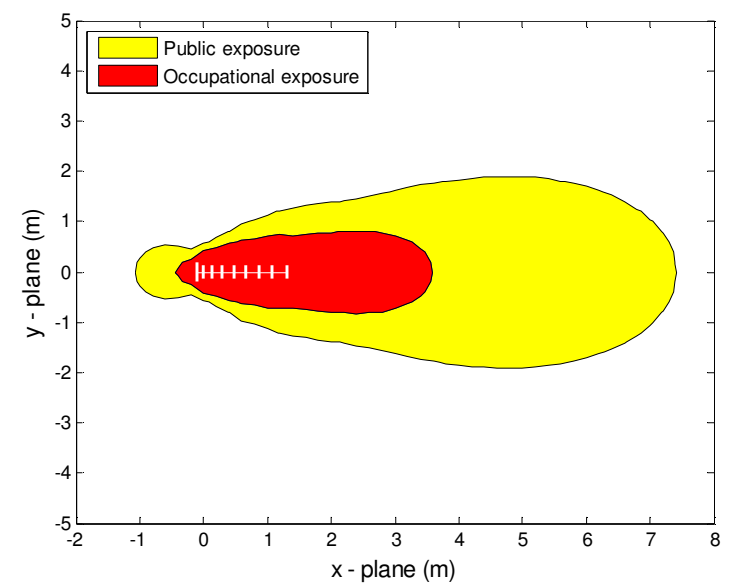

Fig. 3. NEC E-field strength for occupational $(63.6396 \mathrm{~V} / \mathrm{m})$ and general public $(29.1682 \mathrm{~V} / \mathrm{m})$ exposures in $x-y$ plane.

The second tool was ProX5 EME [8], a software developed by Corearth Group based on R.C. Hansen's aperture model [9], for the modelling of electromagnetic emissions. The engine is built upon far-field calculations, near-field gain tapering, aperture-based analysis and boundary analysis.

For the near field calculations, ProX5 EME compensates for the overestimating effect of just using the far field calculations. This is required because the directivity is less in the near field due to the finite size of the aperture. The engine was developed from power density observations for uniform line sources and tapered illumination aperture antennas. The basis was to define a general breakpoint distance where the different calculations are applied for near and far fields and a taper method is used in the transition distance.

In order to validate ProX5 EME, the far field radiation pattern was simulated and compared with the one obtained using NEC. Fig. 4 shows the radiation pattern in polar scale for both horizontal $(x-y)$ and vertical $(x-z)$ planes, using $50 \mathrm{~W}$ transmit power. The front/back ratio shows slightly differences between models, with gains of $11.46 \mathrm{dBi}$ for NEC and $14.15 \mathrm{dBi}$ for ProX5 EME.

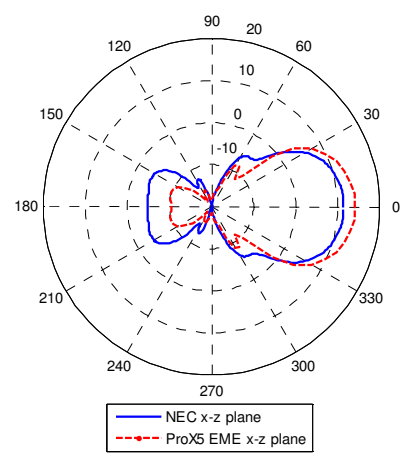

(a)

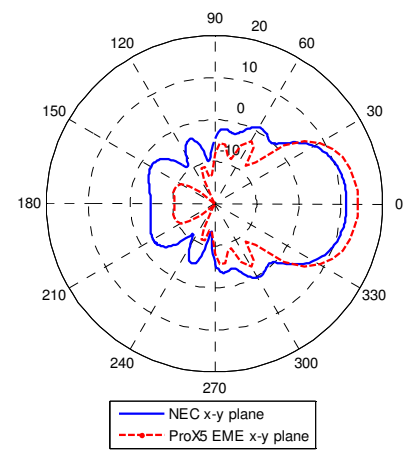

(b)
Fig. 4. NEC and ProX5 EME radiation patterns as a function of the gain in dBi for (a) $x-z$ plane and (b) $x-y$ plane. 
Fig. 5 shows the radiation pattern for both occupational and general public exposures in plan view ( $x-y$ plane) using ProX5 EME.

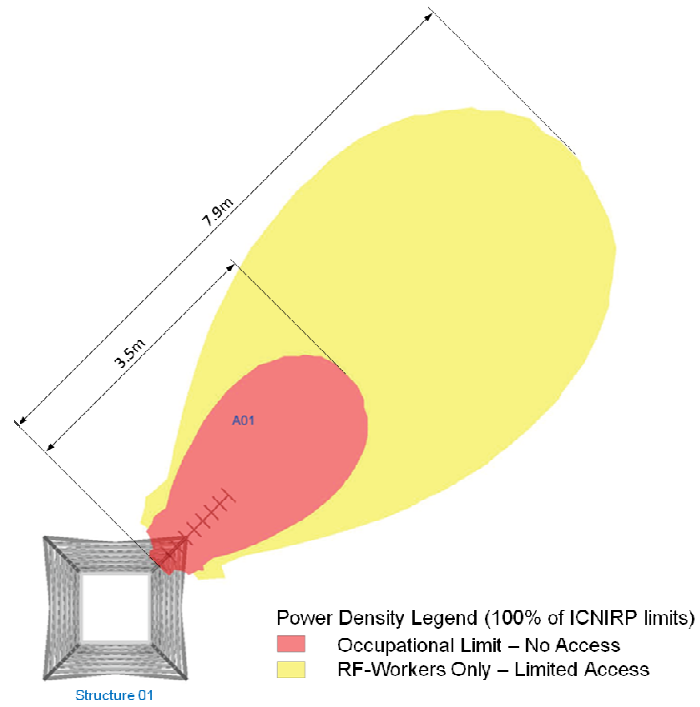

Fig. 5. ProX5 EME occupational and general public field exposures in plan view $(x-y$ plane).

The third tool used was the analytical Cylindrical Wave formulation, which is the model used by CENELEC (European Committee for Electrotechnical Standardization) and it is based on the formulations of the BSI standards [3]. This model does not take into account the radiation pattern and uses only the main beam power level. For the near field region, in a sectorcoverage array, the corresponding prediction equation for the spatially-averaged equivalent power density is defined as

$$
\bar{S}(r, \varphi)=\frac{P_{a v} 2^{-\left(2 \frac{\varphi}{\Phi_{3 d B}}\right)^{2}}}{\Phi_{3 d B} r L \cos ^{2}(\gamma) \sqrt{1+\left(\frac{r}{\frac{\Phi_{3 d B}}{12} D_{A} L \cos ^{2}(\gamma)}\right)^{2}}}
$$

where $\varphi$ is the azimuth angle with a maximum gain when $\varphi=0 \mathrm{rad}, \Phi_{3 \mathrm{~dB}}$ is the azimuth $3 \mathrm{~dB}$ beam width of the radiation pattern (rad) and it was extracted from the RFI manufacturer's technical data sheet [7], $P_{a v}$ is the power at the antenna port $(\mathrm{W}), L$ is the physical antenna length $(\mathrm{m}), D_{A}$ is the peak antenna directivity (unit-less), $\gamma$ is the electrical down-tilt angle of the antenna main beam (rad) and $r$ is the distance from the antenna to the point of investigation (m). Fig. 6 shows the spatial-averaged equivalent power density $\left(\mathrm{W} / \mathrm{m}^{2}\right)$ for sector-coverage arrays, simulated as a function of the azimuth angle $(-\pi<\varphi<\pi)$.

The fourth tool is the Flat-zone method, a European alternative to the CENELEC standard used to determine the zone boundaries from mobile phone base station antennas. The method provides only the occupational exposure limit.
Both the Cylindrical wave model and the Flat zone method provide the zone boundaries measured from the front end of the antenna.

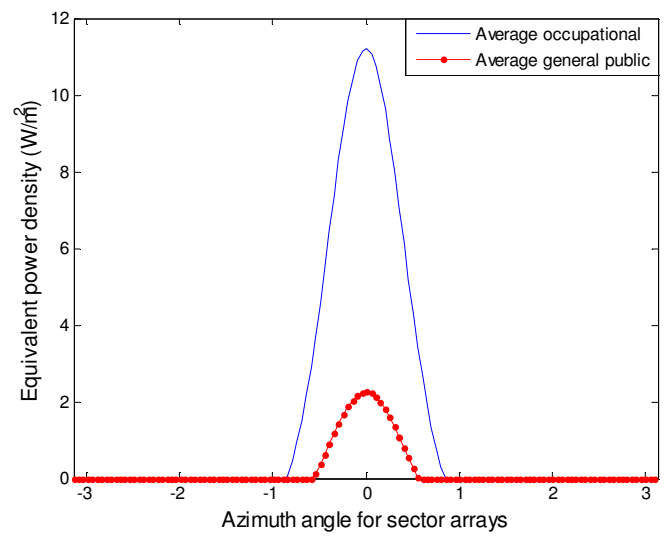

Fig. 6. Occupational and general public spatial-averaged equivalent power density $\left(\mathrm{W} / \mathrm{m}^{2}\right)$ for sector-coverage arrays, as a function of the azimuth angle in the $x-y$ plane, using the cylindrical wave model.

\section{RESULTS}

The 3D radiation pattern for the occupational and general public exposure levels were simulated using NEC, ProX5 EME, Cylindrical wave model and Flat zone method.

Fig. 7 shows a comparison between the four methods described previously as a function of the zone boundary distance $(\mathrm{m})$, measured from the active element (folded dipole) of the antenna in direction to the main beam, to the ICNIRP equivalent plane wave power density levels given in Table I. As the Cylindrical wave model and the Flat zone methods evaluate distances from the end of the antenna, the ranges were recalculated using the position of the active feed element to match the NEC results.

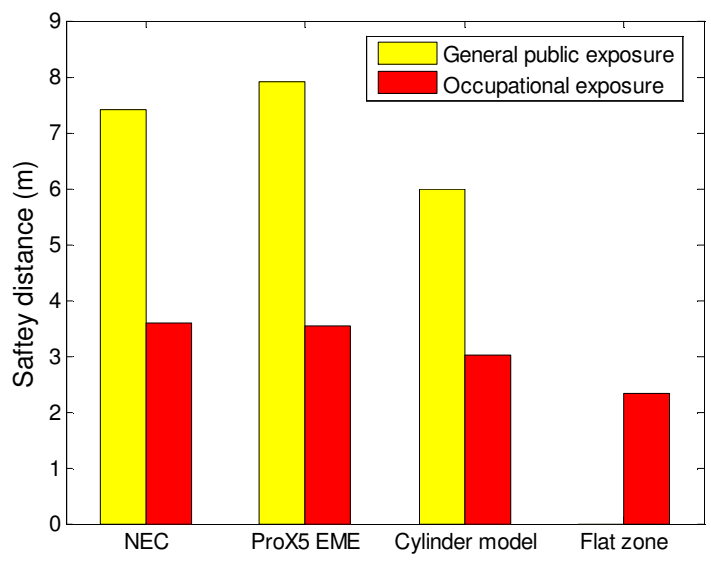

Fig. 7. Comparison between NEC, ProX5 EME, Cylindrical model and Flat zone method, as a function of the distance from the active element of the antenna to the ICNIRP power density levels along the boresight. 
In addition to the occupational and general public exposures measured in the direction of the main beam $\left(\theta=90^{\circ}\right.$ and $\varphi=0^{\circ}$ ), off-axis zone boundaries were extracted from NEC 3D electric and magnetic radiation patterns and are shown in Table II for the three axis $(x, y, z)$ with different angles.

The computations were performed on an Intel Core i5 at $3.20 \mathrm{GHz}$ PC with $4 \mathrm{~GB}$ of RAM. The near-field radiation pattern simulations on NEC were computed in $2 \mathrm{~min}$ for each plane, and in less than 2sec on ProX5 EME. The Cylindrical wave model and the Flat-zone method use only the main beam power level.

TABLE II. OFF-AXIS ZONE BOUNDARIES FROM NEC 3D ELECTRIC AND MAGNETIC RADIATION PATTERNS

\begin{tabular}{|c|c|c|c|c|c|c|c|}
\hline \multicolumn{2}{|c|}{ Angle $\left({ }^{\mathbf{}}\right)$} & \multicolumn{3}{c|}{ Occupational exposure } & \multicolumn{3}{c|}{ General public exposure } \\
\hline $\boldsymbol{\theta}$ & $\boldsymbol{\varphi}$ & $\boldsymbol{x}$ & $\boldsymbol{y}$ & $\boldsymbol{z}$ & $\boldsymbol{x}$ & $\boldsymbol{y}$ & $\boldsymbol{z}$ \\
\hline 160 & 30 & $1.4 \mathrm{~m}$ & $0.8 \mathrm{~m}$ & $4.4 \mathrm{~m}$ & $1.4 \mathrm{~m}$ & $0.8 \mathrm{~m}$ & $4.4 \mathrm{~m}$ \\
\hline 135 & 15 & $4.4 \mathrm{~m}$ & $1.2 \mathrm{~m}$ & $4.6 \mathrm{~m}$ & $4.4 \mathrm{~m}$ & $1.2 \mathrm{~m}$ & $4.6 \mathrm{~m}$ \\
\hline 90 & 0 & $3.6 \mathrm{~m}$ & $0 \mathrm{~m}$ & $0 \mathrm{~m}$ & $7.4 \mathrm{~m}$ & $0 \mathrm{~m}$ & $0 \mathrm{~m}$ \\
\hline 80 & 10 & $3.4 \mathrm{~m}$ & $0.6 \mathrm{~m}$ & $0.6 \mathrm{~m}$ & $6.6 \mathrm{~m}$ & $1.2 \mathrm{~m}$ & $1.2 \mathrm{~m}$ \\
\hline 75 & 15 & $2.2 \mathrm{~m}$ & $0.6 \mathrm{~m}$ & $0.6 \mathrm{~m}$ & $5.2 \mathrm{~m}$ & $1.4 \mathrm{~m}$ & $1.4 \mathrm{~m}$ \\
\hline 60 & 30 & $2.4 \mathrm{~m}$ & $1.4 \mathrm{~m}$ & $1.6 \mathrm{~m}$ & $2.4 \mathrm{~m}$ & $1.4 \mathrm{~m}$ & $1.6 \mathrm{~m}$ \\
\hline 45 & 45 & $1 \mathrm{~m}$ & $1 \mathrm{~m}$ & $1.4 \mathrm{~m}$ & $1 \mathrm{~m}$ & $1 \mathrm{~m}$ & $1.4 \mathrm{~m}$ \\
\hline 30 & 60 & $0.8 \mathrm{~m}$ & $1.4 \mathrm{~m}$ & $2.8 \mathrm{~m}$ & $0.8 \mathrm{~m}$ & $1.4 \mathrm{~m}$ & $2.8 \mathrm{~m}$ \\
\hline
\end{tabular}

Table III shows the absolute error between NEC with ProX5 EME, Cylindrical wave model and Flat zone method, considering NEC as the reference value, with an indication when the values are above (+) or below (-) the NEC ones.

TABLE III. ABSOLUTE ERROR (\%) BETWEEN NEC, PROX5 EME, CYLINDRICAL WAVE MODEL AND FLAT ZONE METHOD

\begin{tabular}{|l|c|c|}
\cline { 2 - 3 } \multicolumn{1}{c|}{} & $\begin{array}{c}\text { Occupational } \\
\text { exposure }\end{array}$ & $\begin{array}{c}\text { General public } \\
\text { exposure }\end{array}$ \\
\hline NEC vs ProX5 EME & $-1.4 \%$ & $+7.4 \%$ \\
\hline NEC vs Cylinder model & $-16 \%$ & $-18 \%$ \\
\hline NEC vs Flat zone method & $-35 \%$ & --- \\
\hline
\end{tabular}

\section{CONCLUSIONS}

This study provided a comparison between NEC, ProX5 EME, Cylinder wave model and Flat-zone method. NEC was considered as the reference value in the analysis. The results showed good agreement in the direction of the main beam (Fig. 7). ProX5 EME proved to be the most accurate of the methods, especially in the occupational exposure limit, where the error obtained was $1.4 \%$ compared to the equivalent NEC results.

The Flat-zone method provided a much lower zone boundary distance $(2.32 \mathrm{~m})$ compared with the other three methods. The NEC field exposure zone patterns differ in the near field but will be identical in the far field.
Polar plots in horizontal and vertical planes from NEC and ProX5 EME were included, in order to verify that generic patterns were employed and to clearly demonstrate the relationship between a log-based gain plot and linear space exclusion zone.

Off-axis distances from NEC were presented in Table II. At some angles, the occupational and public general levels were the same, verifying the directive behavior of the YagiUda antenna. Only at angles close to the direction of the main beam was the difference between occupational and general public exposures considerable. At $\theta=90^{\circ}$ and $\varphi=0^{\circ}$, the resulting distances confirm the calculations obtained in Fig. 7.

As a future work, different types of antennas such as corner reflectors, panels and parabolic dishes will be assessed using the four methods proposed in this paper. Off-axis zone boundaries from ProX5 EME will be calculated.

\section{REFERENCES}

[1] ARPANSA, "Radiation Protection Standard: Maximum Exposure Levels to Radiofrequency Fields - $3 \mathrm{kHz}$ to $300 \mathrm{GHz}$," Australian Radiation Protection and Nuclear Safety Agency, Melbourne, RPS 3, May 2003.

[2] IEEE SCC28, "IEEE Recommended Practice for Measurements and Computations of Radio Frequency Electromagnetic Fields with Respect to Human Exposure to Such Fields, $100 \mathrm{kHz}$ - 300GHz," IEEE International Committee on Electromagnetic Safety, New York, IEEE Std C95.3, Dec. 2002.

[3] Basic standard for the calculation and measurement of electromagnetic field strength and SAR related to human exposure from radio base stations and fixed terminal stations for wireless telecommunication systems (110MHz - 40GHz). ISBN: 978058068572 9, BSI 2010.

[4] NEC-Win Pro, Version 1.6.2d Nittany Scientific, 2004.

[5] Standard AS/NZS 2772.2 Human exposure to electromagnetic fields, Part 2: Principles and methods of measurement and computation $(3 \mathrm{kHz}$ to $300 \mathrm{GHz}$ ). ISBN: 978073379857 3, 2011.

[6] Guidelines for limiting exposure to time-varying electric, magnetic and electromagnetic fields (up to $300 \mathrm{GHz}$ ) - International Commission on Non-Ionizing Radiation Protection (ICNIRP). Health Physics Society, 1998.

[7] UHF Directional Yagi Antennas, 400-600MHz, YB9 Series.(www.rfi.com.au).

[8] ProX5 EME Rapid Simulation and Quality Compliance Documentation, Version 2.9. Corearth Group, Australia, 2013.

[9] R.C. Hansen, Microwave Scanning Antennas: Apertures. Vol. 1. Academic Press, 1964. 